\title{
An evaluation of referrer factors for 98,671 referrals made to the West Yorkshire oral surgery managed clinical network over a three-year period
}

\author{
Richard J. Moore, ${ }^{* 1}$ lain Pretty, ${ }^{2}$ Gail Douglas ${ }^{3}$ and Alan J. Mighell ${ }^{1}$
}

\section{Key points}

Describes referrals for a tri-speciality MCN over 36 months with insight into the service demand for oral surgery, oral medicine and maxillofaical surgery in West Yorkshire and highlights referral behaviours showing that $60 \%$ of all referrals are accounted for by $10 \%$ of referrers.
Oral surgery referrals accounted for over $75 \%$ of all referrals of which the majority were exodontia. Almost $20 \%$ of all referrals were in the 'other' category.
Highlights the need for further studies both of a quantitative and qualitative nature to better understand referrer behaviours and how these impact on future service design and workforce training.

\section{Abstract}

Introduction Patients referred from primary dental care to hospital-based specialists in high volumes can contribute to significant NHS service pressures. Surprisingly, little is understood about what contributes to referral factors.

Aims To gain new insight into the referral factors from primary dental care by interrogating the tri-speciality West Yorkshire managed clinical network (MCN) referral pathway data for a 36-month period (2016-2019).

Methods Anonymised referrals from the electronic referral management system were collated for analyses.

Results There were 98,671 referrals within the 36 -month period, $12.3 \%$ of which were rejected. Of those accepted for triage, $76 \%$ were directed at oral surgery, with $>60 \%$ accounted for by exodontia. In total, $10 \%$ of referrers accounted for $60 \%$ of all referrals. Peak referral occurred five years after General Dental Council registration.

Discussion This is the first report of referral data from a tri-speciality MCN with exodontia referrals predominating. The data set demonstrates variation in referrer behaviours despite referral guidance. Referrals should be based upon patient need but patterns observed in this study suggested possible associations with high and low referral patterns which warrant further research.

Conclusions Interrogation of the referral database suggests that there are interesting patterns of referral which may be associated with characteristics of the referrer as well as their patients' needs. Further investigation could inform improved processes and service design, as well as education delivery and workforce development.

\section{Introduction}

The referral of patients from primary dental care to hospital-based specialists is a long-established model in the UK. Over time, referral numbers grew, exposing the limitations of a demanddriven approach within increasing pressure on NHS resources. To help meet this challenge, the NHS England 'Five year forward view'1 set the policy for introduction of managed clinical networks (MCNs). MCNs are an established

${ }^{1}$ Division of Oral Surgery and Oral Medicine, University of Leeds, UK; ${ }^{2}$ School of Dentistry, University of Manchester, UK; ${ }^{3}$ Department of Dental Public Health, University of Leeds Dental School, UK.

*Correspondence to: Richard J. Moore

Email address: r.moore2@leeds.ac.uk

Refereed Paper.

Accepted 10 May 2021

https://doi.org/10.1038/s41415-022-4034-z healthcare model that aim to provide care in a timely fashion at an appropriate location that is delivered by the most suitable professional in the networked area. ${ }^{2}$ In brief, there is greater flexibility in how care is provided compared to the established model of primary care dental team referral directly to hospital specialists, with attendant advantages for patients and NHS services.

NHS England went on to publish the 2015 'Guide for commissioning oral surgery (OS) and oral medicine (OM)'3 to support NHS commissioners to offer a 'consistent and coherent approach' to commissioning services, for example in OS, across three levels of care within an MCN approach. Levels 1 and 3 reflect care delivered by general practitioners and specialist/consultant led services ( $3 \mathrm{a}$ and $3 b)$, respectively. Level 2 care is delivered by dentists with skills over and above those expected of a general dental practitioner who are working within a networked approach that incudes patient pathways. The levels of care are not tied to the setting of the provider and may include primary care or hospital settings.

In 2004, the introduction of the NHS Choose and Book System ${ }^{4}$ in England, an electronic referral management system (eRMS) for general medical practitioners (GMPs) to refer to specialist medical services, ended the traditional paper letter referral in that area of healthcare. It empowered patient choice over service location and gave the referrer more autonomy. ${ }^{5}$ The eRMS has been associated with a reduction in inappropriate referrals, waiting times and duplicate referrals. ${ }^{5}$ In addition, patient satisfaction increased. This represented a step change with subsequent eRMS referral development, in part informed by the data that digitalisation generated. 
By contrast, dentistry largely continued with a paper-based referral process. However, there has been an increasing switch to eRMS in dentistry over recent years, but the pace, prioritisation and system choice has been in response to local or regional drivers that contrasts with the NHS e-Referral system used universally across England by GMPs. This difference is due to the central funding of general practioner IT systems, whereas other primary care providers, including dentists, were not included in this NHS funding.

Provision of care that falls within the scope of OS, oral and maxillofacial surgery (OMFS) or OM varies across the UK, reflecting how local services have developed over time. These areas of clinical practice collectively account for the majority of all referrals made by the dental team, yet surprisingly, little is understood about referrer factors. New insight has the potential to inform improved processes and service design, as well as education delivery and workforce development.

The West Yorkshire Oral Surgery MCN (WYOS MCN), established in October 2016 has a geographical footprint that is home to 1.3 million people. Given the overlap in clinical practice between OS, OMFS and OM, the WYOS MCN established a referral form for each one, but manages these through a single eRMS. The aim of this study is to gain insight in to the referral factors which are contributory to the pathway, by interrogation of the WYOS MCN referral pathway data for a 36-month period (2016-2019).

\section{Materials and methods}

\section{Referral system}

All referrals for the WYOS pathway since its inception on 1 October 2016 have been made through a single eRMS, which is summarised in Figure 1 and additionally by MontgomeryCranny et al. $2017 .^{6}$

Potential referrers are required to make decisions that are informed by referral guides and related documentation to promote best use of the processes. Clear direction is given that suspected cancer referrals should be referred via the two week wait pathway and not via this eRMS. Within the WYOS

\section{Fig. 1 Overview of the WYOS pathway}

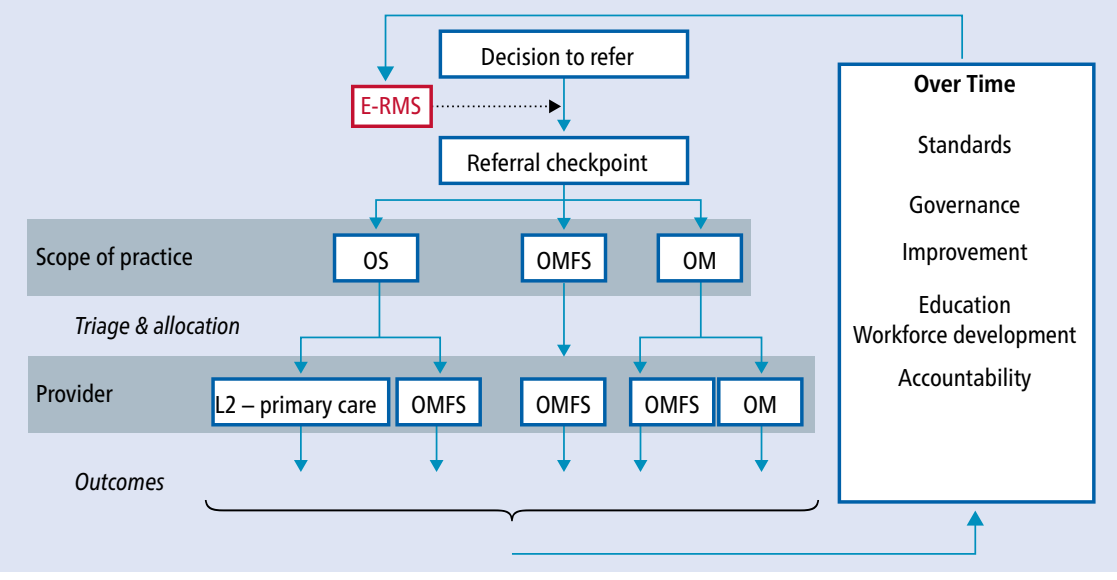

pathway, referrers are required to choose one of three referral forms; OS, OMFS or OM (Appendices 1, 2 and 3). Each referral form includes a structured approach for the clinical focus of the referral and is designed to acquire a minimum data set through the use of mandatory questions. Each referral form includes an 'other' category for conditions that do not fit one of the named categories. As part of the referral process, the referrer in liaison with the patient is asked to select a preferred provider with choice informed by 'nearest' or 'soonest' options.

After receipt, each referral is checked for completeness of essential administrative details with referrals returned to the referrer if these are incomplete. Referrals that meet the required administrative standards are triaged either centrally (OS) or by the referrer-selected local provider (OMFS and OM). Triage takes account of the sufficiency of clinical information to make an informed decision and appropriateness for the service in question. After central triage, allocation of accepted OS referrals to providers is determined by referrer/patient choice and capacity within the overall MCN. It is important to note that the choice of referral form does not determine the provider. For example, care that follows a referral made on an OS form is often provided by OMFS.

\section{Referral data}

Referral data for the three specialties OM, OMFS and OS were acquired from the eRMS provider for the dates October 2016 to September 2019 inclusive. Agreement was sought from the commissioners and WYOS MCN. Ethical approval was not required as this is a service evaluation with secondary use of data, as stated by the Information Commissioner's Office. There

Table 1 Summary of referrals received for clinical triage over the 36-month period summarised by 12 month periods and the three referral forms used

\begin{tabular}{l|l|l|l|l|l|l|l|l|l|}
\multirow{2}{*}{$\begin{array}{l}\text { Time } \\
\text { period }\end{array}$} & \multicolumn{2}{|c}{ Oral surgery } & \multicolumn{2}{c}{ Oral medicine } & \multicolumn{2}{c}{ Oral and maxillofacial } & \multicolumn{3}{c}{ All referrals } \\
\cline { 2 - 10 } & Total received & $\begin{array}{l}\text { Total (\%) } \\
\text { rejected }\end{array}$ & Total received & $\begin{array}{l}\text { Total (\%) } \\
\text { rejected }\end{array}$ & Total received & $\begin{array}{l}\text { Total (\%) } \\
\text { rejected }\end{array}$ & Total received & $\begin{array}{l}\text { Total (\%) } \\
\text { rejected }\end{array}$ \\
\hline $2016-2017$ & 21,550 & $3,171(14.7)$ & 3,872 & $336(8.6)$ & 3,936 & $589(14.9)$ & 26,358 & $4,096(15.5)$ \\
\hline $2017-2018$ & 23,383 & $3,351(14.3)$ & 4,564 & $290(6.3)$ & 3,509 & $261(7.4)$ & 31,456 & $3,902(12.4)$ \\
\hline $2018-2019$ & 29,918 & $3,807(12.7)$ & 4,668 & $207(4.4)$ & 3,271 & $186(5.6)$ & 37,857 & $4,200(11.0)$ \\
\hline Totals & $\mathbf{7 4 , 8 5 1}$ & $\mathbf{1 0 , 3 2 9 ( 1 3 . 7 )}$ & $\mathbf{1 3 , 1 0 4}$ & $\mathbf{8 3 3 ( 6 . 7 )}$ & $\mathbf{1 0 , 7 1 6}$ & $\mathbf{1 , 0 3 6 ( 9 . 6 )}$ & $\mathbf{9 8 , 6 7 1}$ & $\mathbf{1 2 , 1 9 8 ( 1 2 . 3 )}$ \\
\hline
\end{tabular}


were no patient-identifiable data within the spreadsheet. These data were exported from the eRMS into a Microsoft Excel (Microsoft Corporation, 2018) spreadsheet and cleansed into the speciality-specific categories as detailed in the results section.

In addition to this, the numbers of new registrants each year were acquired from the General Dental Council (GDC) and plotted against the years since first registration of the local referrers; referral numbers for each referrer were formatted into deciles.

\section{Results}

Total number of referrals received for clinical triage over 36 consecutive months

A total of 98,671 referrals were made through the eRMS over the 36-month study period starting 1 October 2016 (Table 1).

Of these, $12.3 \%(n=12,198)$ were rejected at clinical triage due to reasons such as no radiographs, inappropriateness of referral and insufficient clinical details. Fluctuations in the number of referrals received per month across the 36-month study period were evident, as shown in Figure 2, that started in October 2016 (month 1) with the introduction of the WYOS MCN. Transient reductions are evident in August (months 11, 23 and 35) and December (months 3, 15 and 27).

Each referral form requires information about the reason for referral within several broad categories. There was a wide distribution in the numbers of referrals made within each referral category (Table 2).

Distribution of referrers by year since GDC registration and primary registrable qualification

A total of 1,800 individual referrers made a referral that was accepted for clinical triage. Of these, 3.5\% $(n=63)$ had an invalid GDC number and accounted for 64 referrals. Consideration of the number of referrals made by years since GDC registration identified that the peak for referrals was five years after GDC registration and thereafter fell (Fig. 3). UK graduates made $75 \%$ of all referrals with the remainder by overseas graduates.

A regression analysis was carried out on the distribution of referrers, years since first registration and referral numbers which showed no significant F-test and no significant cofficients; therefore, the numbers of years since graduation is not signiciant in the referral
Fig. 2 Referrals received for clinical triage by month over the 36-month period

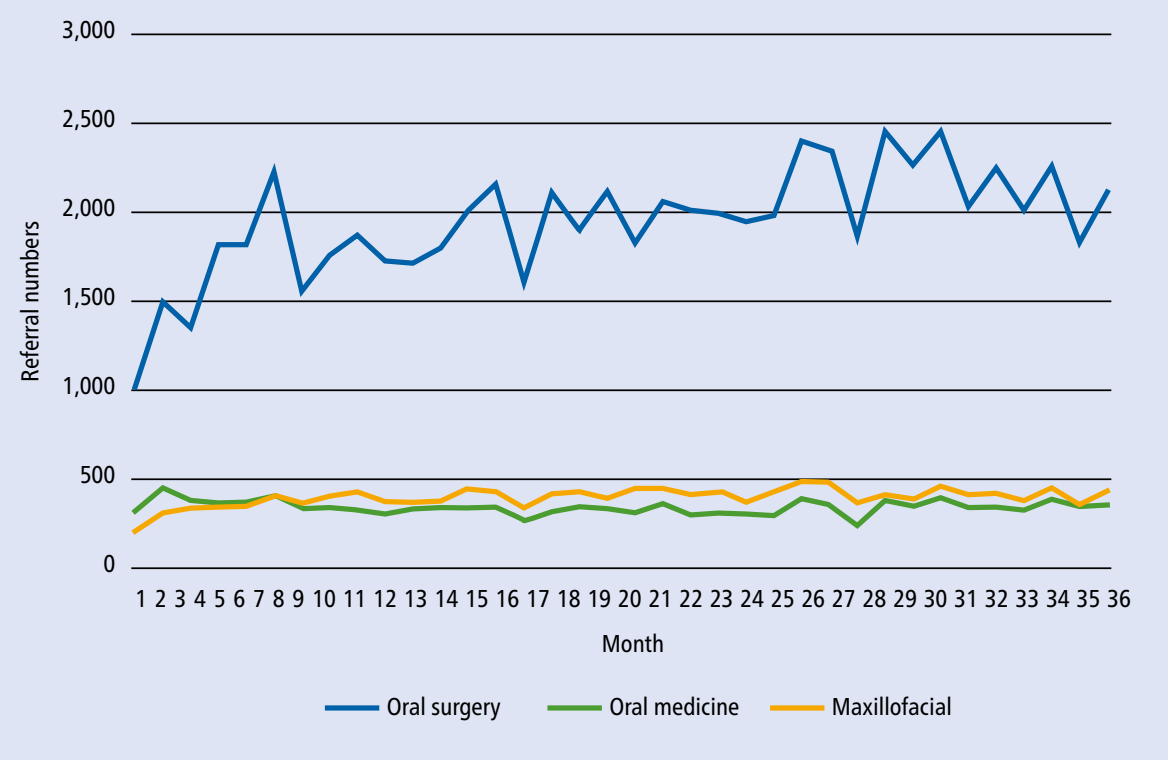

Table 2 Cause for referral, as selected by the referrer, by 12-month period. For each form, the referral categories are presented in order of descending selection apart from the 'other' choice, which is presented last

\begin{tabular}{|c|c|c|c|c|c|}
\hline Form & Referral category & 2016-2017 & 2017-2018 & 2018-2019 & Totals (\%) \\
\hline \multirow{8}{*}{ OS } & Difficult extraction & 6,252 & 6,001 & 4,490 & $16,743(16.9)$ \\
\hline & Third molars & 4,101 & 4,929 & 3,573 & $12,603(12.7)$ \\
\hline & Removal of roots & 3,850 & 4,923 & 7,649 & $16,422(16.6)$ \\
\hline & Routine extraction & 3,516 & 3,518 & 2,689 & $9,723(9.8)$ \\
\hline & Impacted teeth & 1,962 & 2,029 & 1,395 & $5,386(5.4)$ \\
\hline & Surgical exposure & 309 & 447 & 277 & $1,033(1.0)$ \\
\hline & Surgical endodontics & 243 & 165 & 71 & $479(0.4)$ \\
\hline & Other (OS) & 1,317 & 1,371 & 9,774 & $12,462(12.6)$ \\
\hline \multirow{5}{*}{$\mathrm{OM}$} & Soft tissue (OM) & 2,829 & 3,506 & 2,271 & $8,606(8.7)$ \\
\hline & Orofacial pain & 316 & 382 & 293 & $991(1.0)$ \\
\hline & Salivary gland (OM) & 126 & 134 & 99 & $359(0.3)$ \\
\hline & Oral presentation of wider problem & 56 & 72 & 51 & $179(0.1)$ \\
\hline & Other (OM) & 545 & 470 & 1,954 & $2,969(3.0)$ \\
\hline \multirow{8}{*}{ OMFS } & Soft tissue (OMFS) & 829 & 890 & 578 & $2,297(2.3)$ \\
\hline & Temporomandibular joint & 738 & 870 & 16 & $1,624(1.6)$ \\
\hline & Hard tissue & 545 & 487 & 399 & $1,431(1.4)$ \\
\hline & Trauma & 78 & 76 & 55 & $209(0.2)$ \\
\hline & Salivary gland (OMFS) & 78 & 66 & 51 & $195(0.1)$ \\
\hline & Facial deformity & 32 & 22 & 19 & $73(0.07)$ \\
\hline & Severe swelling & 9 & 51 & 10 & $70(0.07)$ \\
\hline & Other (OMFS) & 1,614 & 1,035 & 2,142 & $4,791(4.8)$ \\
\hline \multicolumn{2}{|l|}{ Total } & 27,558 & 31,480 & 34,047 & $98,671(100.0)$ \\
\hline
\end{tabular}


numbers. Figure 3 shows that the number of WYOS MCN referrers mirrors that of the UK picture of the numbers of new dentists registered (data acquired from the GDC).

\section{Numbers of referrals made by individual GDC registrants}

There was wide variation in the number of referrals made by each GDC registrant,with $10 \%$ of all referrers referring $60 \%$ of the total number of referrals in the three-year period (Fig. 4). The most frequent referrers (top $10 \%$ of referrers $[n=180]$ ) followed a similar distribution to the overall cohort, with UK graduate registrants accounting for $77 \%$.

\section{Discussion}

Better patient access and better resource use are at the heart of the NHS England 'Five year forward view'1 and the COVID-19 pandemic from 2020 is sharpening the focus. There are challenges associated with how routine NHS care is most effectively recovered. This aligns with the need to reduce patients visiting hospitals for care which could be delivered in other settings, closer to patients' homes. The pandemic has also highlighted the importance of preparing the workforce to meet the changing oral health needs of patients and services. $^{\text {? }}$

This is the first report of an MCN with an eRMS that collectively includes referrals made to OS, OMFS and OM specialties by the dental team. The establishment of this MCN coincided with the introduction of the first eRMS in the region and provides powerful opportunities to inform planned changes to service design and educational development of the workforce with the goal of improving patient care. The eRMS is used by primary care dental referrers and referrals from others, such as general medical practice teams and hospital-based specialists, come via other routes. Accordingly, the data presented are representative of the vast majority of all referrals received, but not all.

Referral categories in the three referral forms used brings insight into service demand. This is of benefit for referral categories with high and low referral numbers. Numbers are small for some categories such as facial deformity and facial pain; the care pathways are multidisciplinary. Duplication of complex services needs to be avoided and it is important that patients are able to access these services in a timely manner. By contrast, care for referral categories with high numbers is delivered by

\section{Fig. 3 The distribution of referrers by year since GDC registration}

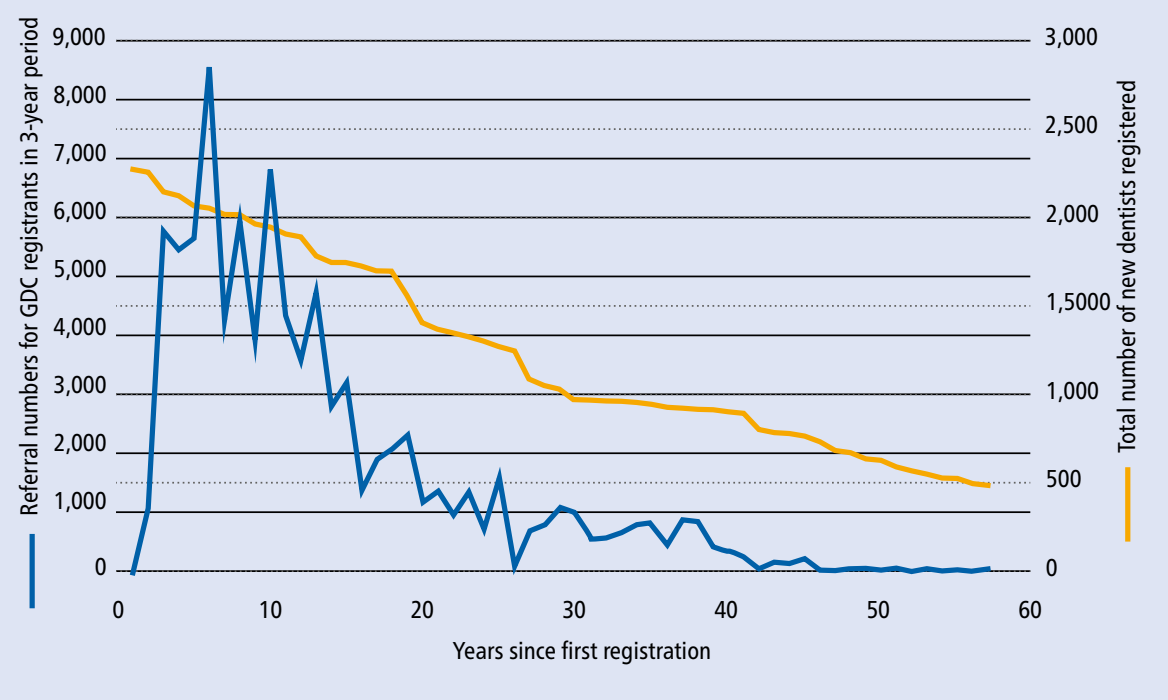

Fig. 4 Numbers of referrals made by individual GDC registrants in deciles

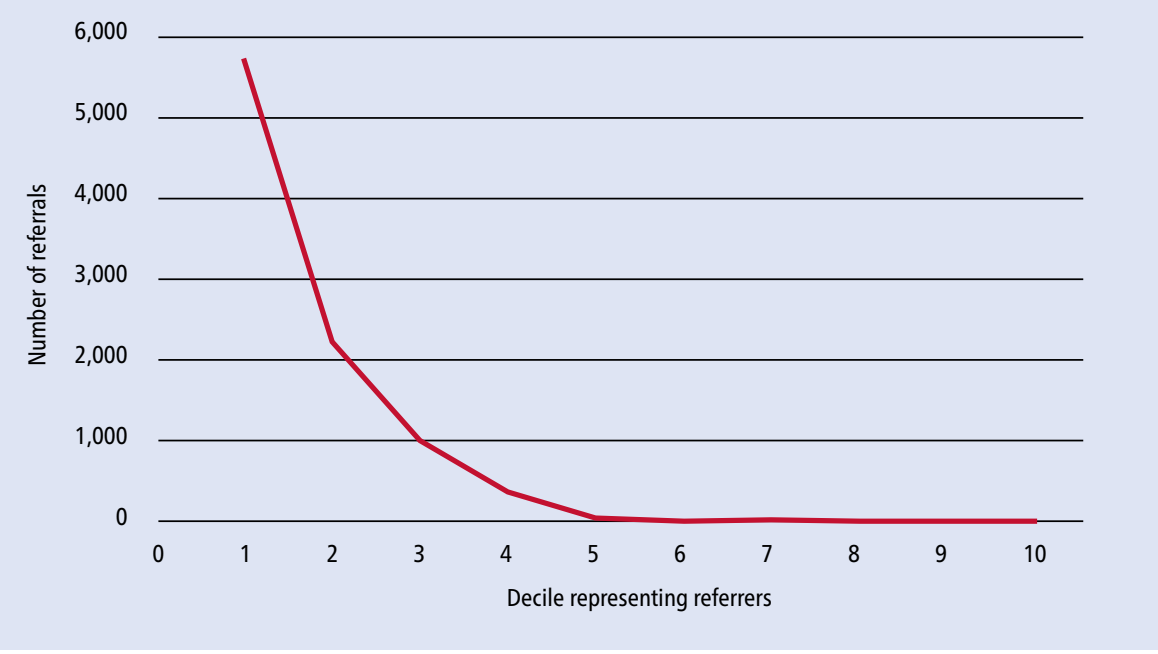

multiple providers who should be delivering care to agreed, minimum standards and shared quality assurance processes. Exodontia in one form or another accounted for over $60 \%$ of all referrals and is likely to be an underestimation given the greater than expected use of the 'other' category. This is consistent with past reports that have highlighted the high referral numbers for Level 2 or 3 OS, including the Getting It Right First Time report for OMFS. ${ }^{8}$

The findings of this study indicate a need to review the referral guidelines and referral categories, as well as how these are used by referrers to ensure there is clarity over which referral form and referral category should be selected. The number of referrals rejected at clinical triage was high at $12.3 \%$ and creates additional work and patient care delays. A further driver for change is evident from the choice of 'other' for all specialties and the rise in its use over the three-year period. Without further qualitative analyses, the appropriateness of such referrals cannot be assessed. It would suggest that either referrals do not fulfil the criteria for the specified categories, or there is scope for more proactive feedback to referrers to limit the use of 'other'. The 'other' category referrals dilute the value of a referral category approach and are a regression to the past paper letter referral approach. The use of 'open text' boxes on referrals provides an opportunity for additional information but only once a specific referral criterion has been chosen.

The variation in referral numbers by year since GDC registration is striking and $60 \%$ of all referrals are accounted for by $10 \%$ of referrers. Within the top $10 \%$, there appears to be no immediate correlation in the years since GDC 
registration and number of referrals, with 77\% $(n=138)$ of the top $10 \%$ of referrers $(n=180)$ being UK graduates. Overall, the percentage of referrers with a UK primary dental qualification was similar (75\%) to the 2019 GDC data for all registered dentists (72\%). ${ }^{9} \mathrm{~A}$ small number of referrals could not be linked to a GDC number but were otherwise valid. These did not have a meaningful impact on data interpretation. Therefore, in this study, referral numbers do not correlate with an 'early years' referrer or overseas graduates. It is important to note that it is not years since graduation and the data must be interpreted accordingly, particularly for overseas registrants who may have worked for many years before registering to work in the UK.

This study includes the largest number of referrals reported for dentistry with recognition that not all referrers would have been working for the whole three-year period and some findings may represent chance. The medical literature reveals a mathematical model which calculates the amount of variation in the rates of referral is likely to be caused by chance alone. ${ }^{10}$ Although this model is applicable to GMPs, the larger size of their referral base compared to that investigated here limits its utility. The heterogeneity of the data presented contrasts with that of Coulthard ${ }^{11}$ who reported that $96 \%$ of a sample of 400 GDPs in one city referred up to ten patients per month for OS care. In this study there is a subgroup of referrers who make high numbers of referrals. This group is of particular interest for further investigation to understand the reasons why. The reasons for this diversity of referral behaviour are likely to be multifactorial, reflecting factors linked to the individual registrant, the running of the practice, location of practice and patient-related factors. However, it is important not to draw unfounded conclusions that their referral patterns are inappropriate. By contrast, some GDC registrants make very few referrals and it is reasonable to question the reasons for this also.

The reasons for the high service demand for exodontia in this referral series need to be better understood. There is a body of published work that explores the self-reported confidence of registrants and included reports that new foundation trainees have low confidence levels with regards to OS. ${ }^{12,13}$ There has been a related focus on the undergraduate and dental foundation training in OS and whether this is sufficient. It might be expected that referral numbers would peak in the years immediately following completion of foundation training, one year after first GDC registration for UK graduates. This is not the case in this large series and the peak at five-years post GDC registration and the subsequent gradual reduction cannot be currently clearly explained. It is probable that the underlying reasons are multifactorial and care has to be taken to not assume that this can be attributed to variations in undergraduate and early career experiences over time. It has been reported that postgraduate training in OS, either as a short course or recognised post-graduate training programme, is a factor contributing to referral behaviours in OS. ${ }^{11}$

The data presented illustrate the value of collectively managing referrals made on OS, OMFS and OM electronic referral forms and how these link to the care provider. Furthermore, it highlights the potential for this to be strengthened by further developing resources and processes to support choice about selection of referral categories, alongside additional patient pathway development.

\section{Conclusions}

In conclusion, this study highlights the potential for three-speciality eRMS data to inform future provision of patient care through improved processes, service design and workforce development. However, further qualitative and quantitative investigation of this data is required. In particular, there is a need to better understand the referral factors which contribute to such high OS referrals of which the majority are exodontia.
Ethics declaration

The authors declare no conflicts of interest.

\section{Author contributions}

The main author is Richard Moore, with equal contributions from Gail Douglas and Iain Pretty. Alan Mighell is the supervising and advisory author for the group.

\section{References}

1. NHS England. Five Year Forward View. 2014 Available at https://www.england.nhs.uk/wp-content/ uploads/2014/10/5yfv-web.pdf (accessed February 2022).

2. Skipper M. Managed clinical networks. Br Dent J 2010; 209: 241-242.

3. NHS England. Guide for Commissioning Oral Surgery and Oral Medicine Specialties. 2015. Available at https://www.england.nhs.uk/commissioning/ wp-content/uploads/sites/12/2015/09/guid-commsoral.pdf (accessed February 2022).

4. Walford S. Choose and Book. Clin Med (Lond) 2006; 6: 473-476.

5. Goldthorpe J, Walsh T, Tickle M et al. An evaluation of a referral management and triage system for oral surgery referrals from primary care dentists: a mixedmethods study. Health Ser Deliv Res 2018; DOI: 10.3310/ hsdr06080.

6. Montgomery-Cranny J, Edmondson M, Reid J, EapenSimon S, Hegarty A M, Mighell A J. Development of a managed clinical network in oral medicine. Br Dent J 2017; 223: 719-725.

7. Health Educatiuon England. Advancing Dental Care Review. 2019. Available at https://www.hee.nhs.uk/ our-work/advancing-dental-care (accessed February 2022).

8. Fullarton M, Martin I, Begley A, Magennis P. Getting It Right First Time (GIRFT) - the Oral \& Maxillofacial Surgery Report: what happens next? Br J Oral Maxillofac Surg 2019; 57: 393-394.

9. General Dental Council. Annual Report and Accounts 2019. 2020. Available at https://www.gdc-uk.org/docs/ default-source/annual-reports/gdc-annual-report-andaccounts-2019.pdf (accessed February 2022).

10. Moore A T, Roland M O. How much variation in referral rates among general practitioners is due to chance? BMJ 1989; 298: 500-502

11. Coulthard P, Kazakou I, Koron R, Worthington H V. Referral patterns and the referral system for oral surgery care. Part 1: General dental practitioner referral patterns. Br Dent J 2000; 188: 142-145.

12. Patel J, Fox K, Grieveson B, Youngson C C. Undergraduate training as preparation for vocational training in England: a survey of vocational dental practitioners' and their trainers' views. Br Dent J 2006; DOI: 10.1038/sj.bdj.4814067.

13. Brown J, Baker R, Moore R. An investigation into the oral surgery curriculum and undergraduate experience in a sample of DFT's in the UK. Eur J Dent Educ 2021; 25: 421-434. 


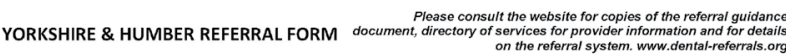

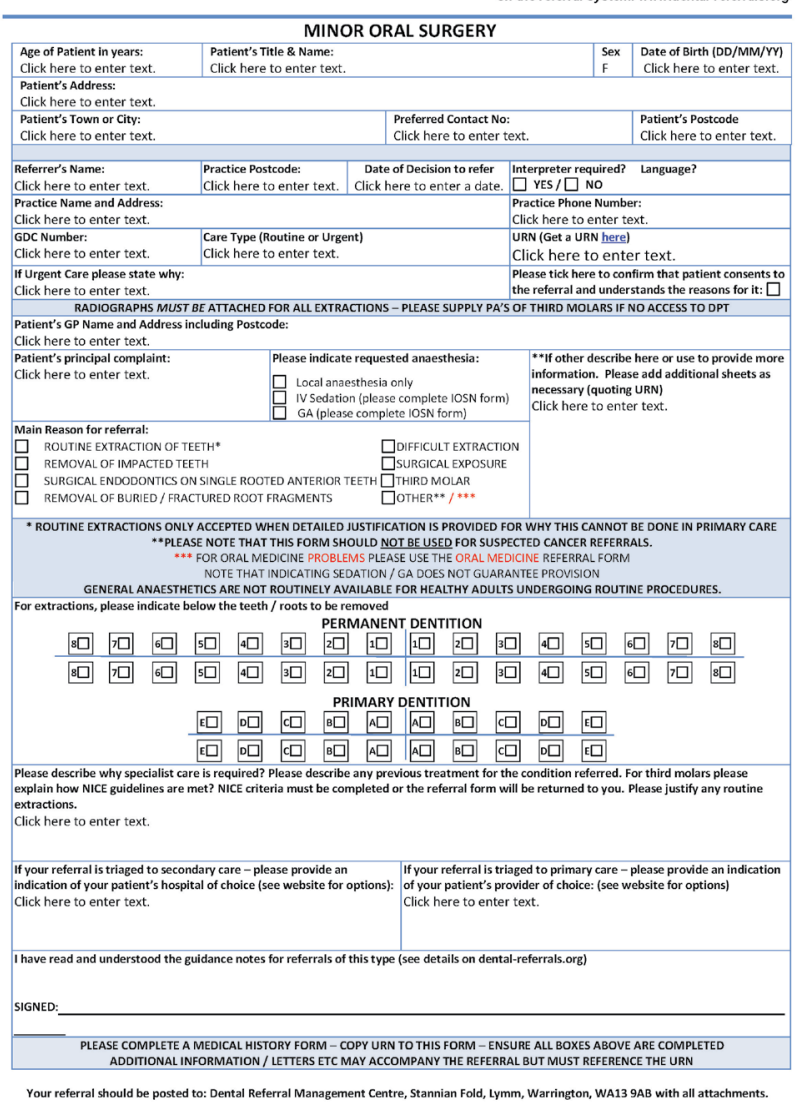

\section{Appendix 1 Oral surgery referral form}

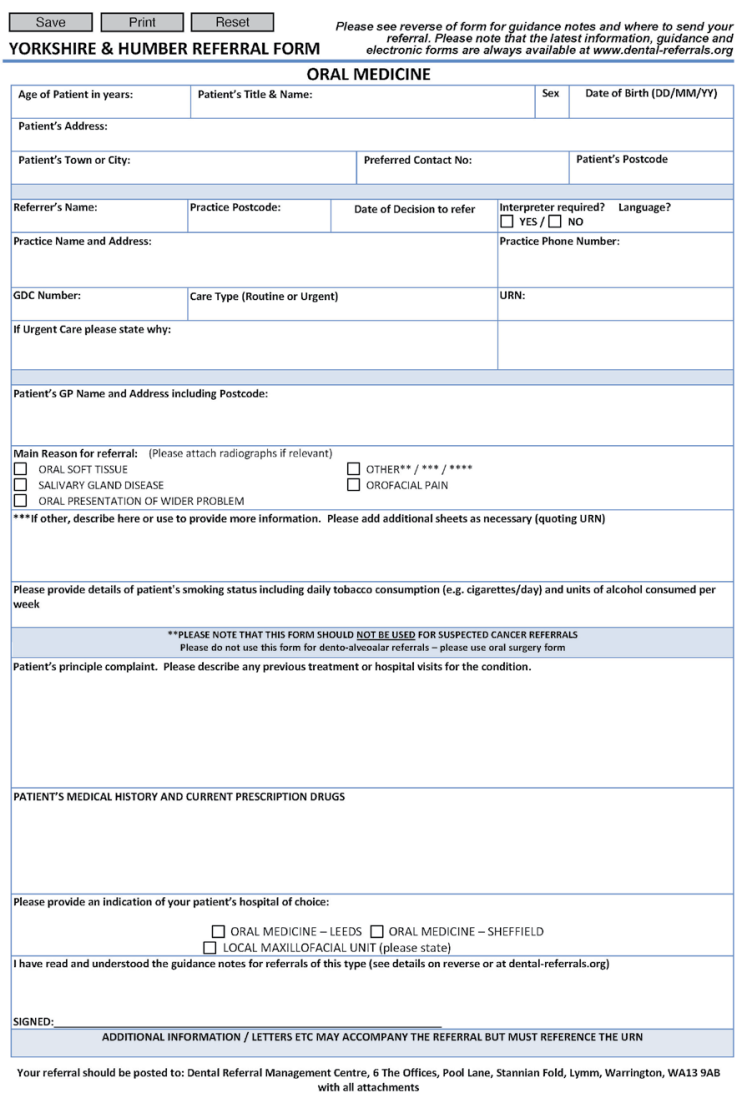

Appendix 3 Oral medicine referral form

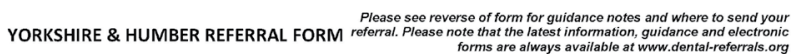

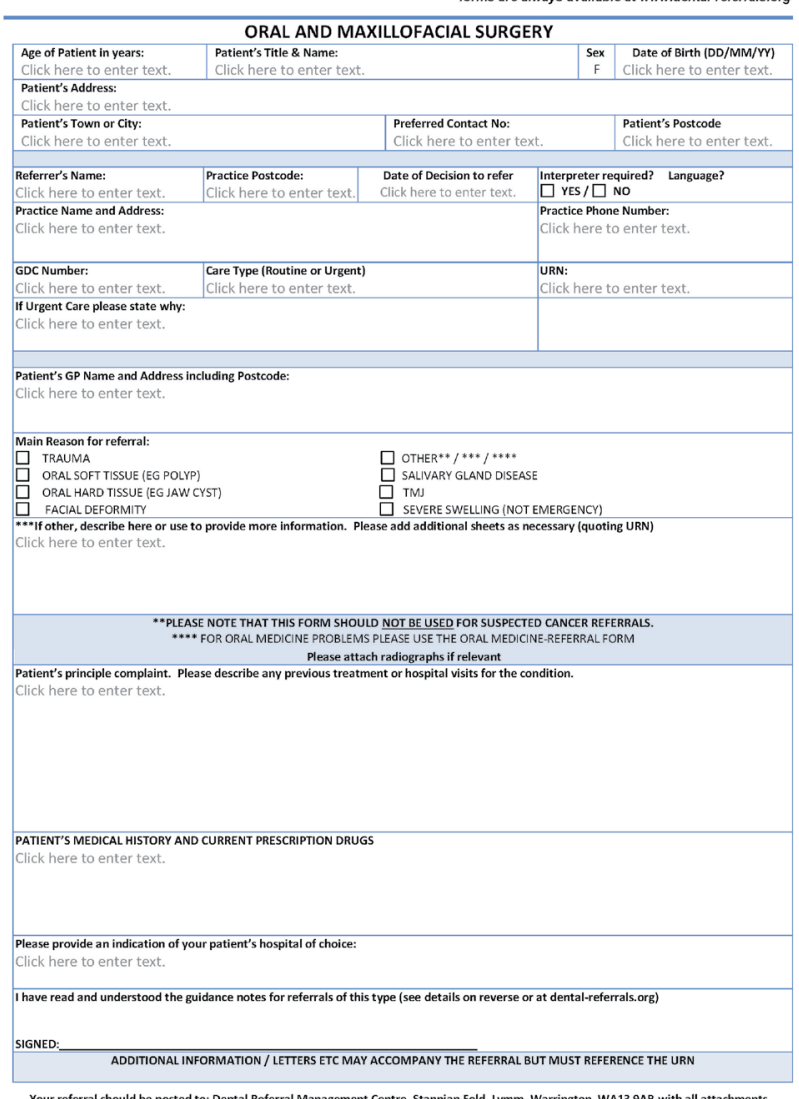

Appendix 2 Oral and maxillofacial surgery referral form 